\title{
MEASUREMENT OF THE UCLA/URLS/INFN HYBRID GUN*
}

\author{
B. O'Shea ${ }^{\dagger}$, A. Boni, A. Fukasawa, J.B. Rosenzweig, University of California, Los Angeles \\ D. Alesini, M. Ferrario, B. Spataro, INFN/LNF, Frascati (Roma), Italy \\ L. Ficcadenti, A. Mostacci, L. Palumbo, Sapienza Università di Roma
}

\begin{abstract}
The hybrid photoinjector is a high current, low emittance photoinjector/accelerator that is under design with collaboration from Roma University La Sapienza, INFN - Laboratori Nazionali di Frascati and the UCLA Particle Beam Physics Lab. The hybrid standing wave-traveling wave photoinjector uses a coupling cell to divide power between a high gradient 1.6 cell standing wave photoinjector, for electron emission and collection, and a lower gradient traveling wave accelerator, for acceleration to desired energies at low emittances. Simulation results show promising beam properties of less than $4 \mathrm{~mm}$-mrad emittance, energy spreads of 1.5 percent, and currents as high as $1.2 \mathrm{kA}$ at energies of $21 \mathrm{MeV}$. We report on the progress of RF design and results of cold test RF measurements at the UCLA Pegasus Laboratory, including methods for measurements and difficulties arising in the transition from simulation to physical measurements.
\end{abstract}

\section{INTRODUCTION}

The hybrid standing wave-traveling wave gun is a system designed for use in present laboratory research or for future implementation where a unit simpler than some of today's standard split gun and accelerators systems is desired. The simplicity of the system arises from the all-in-one philosophy, that is one only needs a radio frequency $(\mathrm{RF})$ source and the system itself and one is ready for experiment. The power division system allows for the omission of RF components like circulators, which are expensive and difficult to produce for higher frequencies such as $\mathrm{X}$-band. The power division system is an appropriately tuned coupling cell and iris which allows the combination of standing wave and traveling wave systems. The initial results are promising as we will show it is possible to maintain a larger field in the standing wave section than in the traveling wave section, while preserving the theoretical phase advance of the system. Beam dynamics simulations can be found elsewhere in these proceedings[1].

\section{RF DESIGN}

The design the of the hybrid system has been split into three sections: the gun, the traveling wave section and the coupling cell. Both the gun and accelerating sections are

\footnotetext{
* Work performed under U.S. Department of Energy contract numbers DE-FG-98ER45693 and DE-FG03-92ER40693

† nadnerbity@gmail.com
}

07 Accelerator Technology Main Systems slight redesigns of current S-band $2.856 \mathrm{GHz}$ models in use throughout the community. The gun is the standard 1.6 cell SLAC/BNL/UCLA design but is fed on axis via a coupling iris instead of the perennial side $\theta$-coupling[2]. The traveling wave section is a $\frac{2 \pi}{3}$ tube based on the design and procedure used by Agostino Marinelli [3]. The most critical piece, the coupling cell, isn't designed separately but in situ in the combined system. The coupling cell is present in all traveling wave structures as an impedance match but in the case of the Hybrid it is also designed to feed power into the standing wave gun[4].

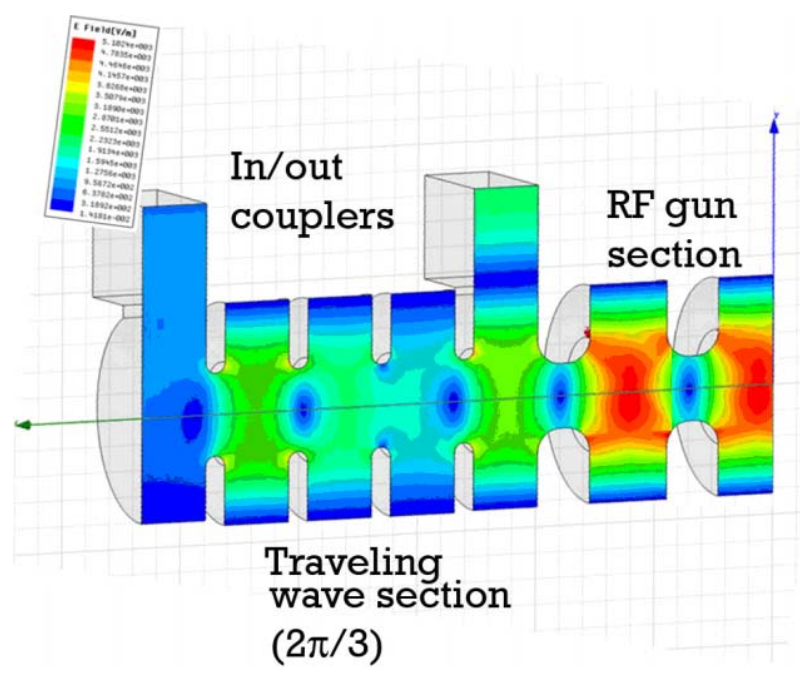

Figure 1: The hybrid gun as simulated in HFSS.

\section{COLD TEST RESULTS}

The Hybrid cold test model is a combined aluminum/copper device designed to include a single period in the traveling wave section using three cells. The device thus contains seven total cells: two copper standing wave cells, three aluminum traveling wave cells and aluminum input and output couplers. It was decided to machine the gun section out of copper due to poor performance of aluminum in previous models. The cells are held together by four steel threaded rods running axial along the device. In order to ensure an adequate mate the rods are placed through the cell-to-cell mating surfaces, this guarantees that the clamping does not cause unnecessary bowing or tension. A non-resonant perturbation method, developed by C.W. Steele, is used for measurement in both the standing wave and traveling wave sections.[5][6].

T06 Room Temperature RF 


\section{Measurement Considerations}

The Slater method for cavity measurement is inadequate for measurement of non-resonant devices and the use of both Slater and Steele methods can make measurements cumbersome and time consuming. In order to apply the Steele method to both SW and TW systems it is advisable to use an extremely small bead, in this case fashioned out of aluminum foil, when measuring resonant cavities. If too large a bead is used the system is thrown wildly out of resonance and the results are disappointing and inaccurate.

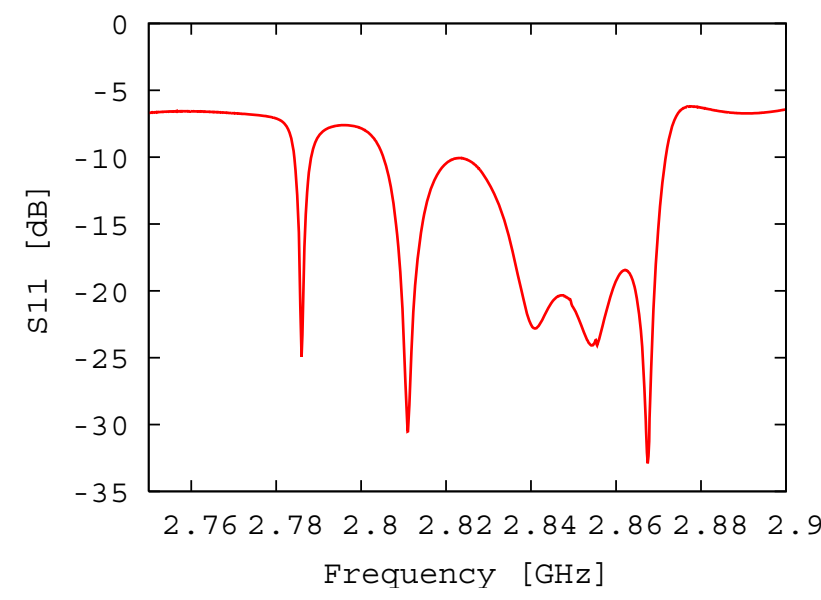

Figure 2: The S11 for the hybrid cold test model.

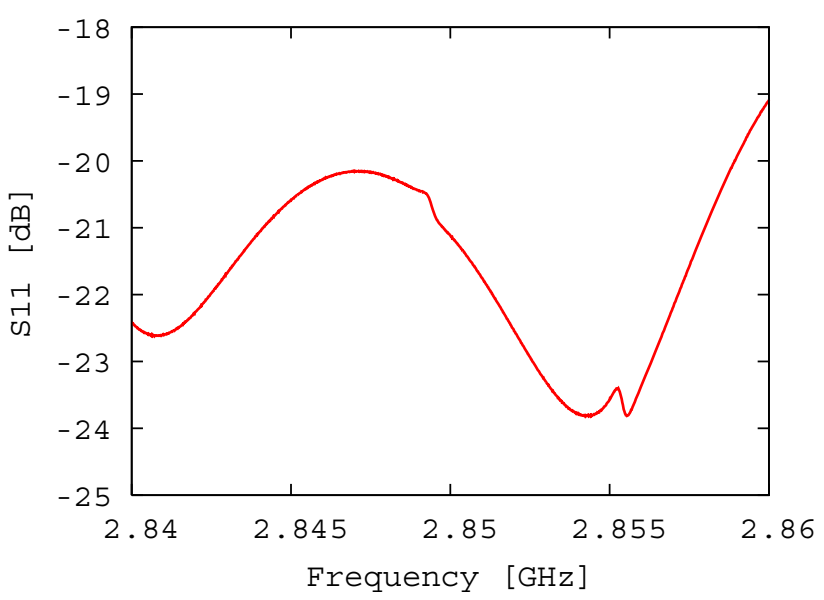

Figure 3: A closer look at the S11 between 2.84 and 2.86 $\mathrm{GHz}$. Clearly seen here are the resonant markings of the standing wave section on the band pass of the traveling wave section. At $2.856 \mathrm{GHz}$ is the $\pi$-mode and at 2.849 $\mathrm{GHz}$ is the 0 -mode.

\section{Tuning Procedure}

The present bead pull system is a simple weight and stepper motor system where the motor rotates and pulls the bead through the cold test model, which requires a hole in the cathode plate. In order to properly measure the electric field on axis the measurement must be made at the correct frequency. The resonant frequencies of the gun are not always visible on the S11 curve and even when they can be seen, they can be ambiguous. In order to measure the pertinent standing wave information a semirigid coaxial line is straightened and a $1-2 \mathrm{~mm}$ piece of the center conductor is exposed to form and antenna. When placed properly in the hole in the cathode plate the transmission from the input port to the antenna gives the frequencies of the 0 and $\pi$ modes as well as mode separation.

\section{Electric Field Measurements}

Tuning screws were added to the standing wave section to counteract imprecise machining, uneven mating and poor clamping through slight variability in cavity frequency. The desired goal of higher fields in the standing wave section than in the traveling wave section can be seen in Figure 1, along with the results from the HFSS simulation.

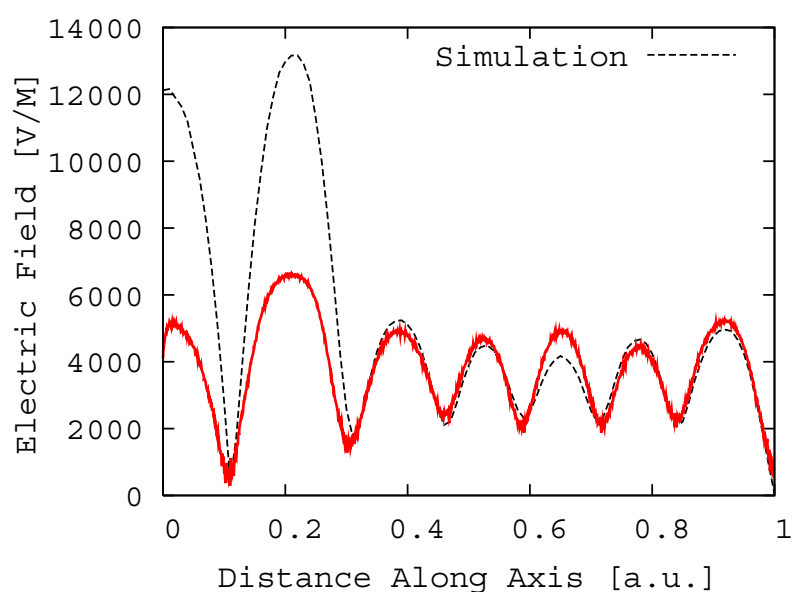

Figure 4: The electric field as measured in the hybrid cold test model. The solid line is the cold test measured value and the dashed line is the simulation result. The electric field has been normalized between the two measurements to clarify the results. The standing wave section is the section running from 0 to 0.3 .

\section{Phase Advance Measurements}

As can be seen in Figure 3, the phase advance between cells is as to be expected as per the simulation results. The seeming zero degree phase change between the first and second cell is a result of the ambiguity between in 0 and $\pi$ in measurement. The electric field can be used to determine the mode in that because the electric field goes to zero between the two cells the device must be operating in the $\pi$-mode. Most importantly the $\frac{\pi}{2}$ phase advance between the second cell and the third cell is evident[4].

T06 Room Temperature RF 


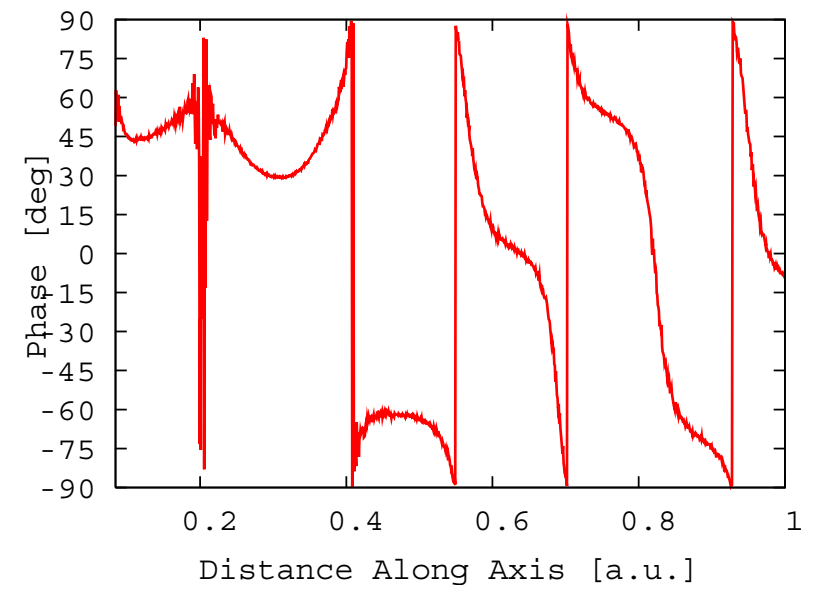

Figure 5: The phase advance as measured in the the hybrid cold test model. The key feature of a $\frac{\pi}{2}$ phase advance between the standing wave section and traveling wave section is evident. The standing wave section is the section running from 0 to 0.4 .

\section{$Q$ Measurements}

The method used for coupling energy into the gun does not lend itself well to the measurement of the unloaded Q of the cavity. While several methods have been used to determine the loaded Q of the cavity, lack of known coupling parameters makes it difficult to obtain the unloaded Q. A small bead to perturb the gun and the Steele method for determining on axis field levels were used to measure a loaded Q, but the results are only interesting in terms of previous values. In this case, perturbation methods are useful as far as determining relative cavity quality between prototype models but not absolute values. Between the two prototypes produced a rise from 4500 (aluminium) to 9000 (copper) was seen in the loaded Q value. While differences in material account in part for the change in $\mathrm{Q}$ value, mate method and machining tolerances also played a roll. An antenna made from a rigid copper wire can also be used to measure the loaded $\mathrm{Q}$ through traditional transmission methods but is also confounded by lack of known coupling parameters and poor repeatability.

\section{CONCLUSION}

Matching is clearly a delicate issue in the production of the hybrid as it determines the division of power between the two systems. As each cold test model becomes more advanced the machining precision and clamping system used in it's construction is taken to more intricate levels and the results improve.

The present design of the hybrid does not include laser ports in the 0.6 cell, thus in order for proper laser illumination of the cathode the original design of a large accelerating section immediately following the gun section must be changed to allow for a gap after $\mathrm{n}$ periods. This gap will allow the fitting of a mirror for reflection of light along the tube's axis. The present bead pull system will not be possible with the final design as there will not be a hole in the cathode. However, the shorter initial section of the hybrid gun will allow for bead drops and avoid the problem of controlling line deflection over the course of 3 meters.

Present considerations for fabrication of the hybrid are a standard braze for the gun and attached traveling wave section and a moving braze for the longer downstream acceleration section. For the gap between sections a simple waveguide, of correct phase length, can be used to transfer power from one section to the other.

Future plans include a high power model and a dual input coupling scheme. Most importantly the next step is to devise a method for reliable and repeatable $\mathrm{Q}$ measurement of the gun section. Also planned is an attempt to scale the device to X-Band, to take advantage of better beam properties especially at low charge, which is made possible by the lack of RF peripherals.

\section{REFERENCES}

[1] A. Fukasawa et al., "Charge and Wavelength Scaling of the UCLA/URLS/INFN Hybrid Photoinjector," these proceedings.

[2] D.T. Palmer et al., "Microwave Measurements of the BNL/SLAC/UCLA 1.6 Cell Photocathode RF Gun," SLACPUB-95-6799.

[3] A. Marinelli, "Progeto di un Accoppiatore in Guida d'Onda per una Struttura Accelerante ad Onda Viaggiante in Banda X," Thesis, June 2004.

[4] B. O'Shea et al., "RF Design of the UCLA/INFN/URLS Hybrid SW/TW Photoinjector,' AAC'06, July 2006, Lake Geneva, WI.

[5] C.W. Steele, "Nonresonant Perturbation Theory," IEEE Trans. on Microwave Theory and Techniques, Vol. MTT-14, No. 2,1966,pp.70-74.

[6] D.V. Land, "Measurement of radio-frequency and microwave fields by nonresonant perturbation," IEE Proc, Vol 131, Part II, February 1984. 